\title{
Magnetic and Electric Signals Precursory to Earthquakes: An Analysis of Japanese Data
}

\author{
Tsuneji RIKITAKE \\ Department of Earth Sciences, College of Humanities and Sciences, Nihon University, Setagaya-ku, \\ Tokyo, Japan
}

(Received June 10, 1986)

\begin{abstract}
Magnetic and electric data of earthquake precursor amounting to 61 in number so far obtained in Japan are analyzed. The relations between main shock magnitude $(M)$, precursor time $(T)$ and epicentral distance $(D)$ are studied for precursors of five subdisciplines $g$ (geomagnetic field change), earth currents $(e)$, resistivity measured by electrodes having a long distance interval $(R)$, resistivity measured by a resistivity variometer $(r)$ and electromagnetic radiation $(w)$.

Linear relations between $\log T$ and $M$ are obtained for subdisciplines $g, e$ and $R$. $T$ for the latter two subdisciplines is smaller than that for subdiscipline $g$ for a fixed $M$. Such a difference can be understood by taking stress build-up and dilatancydiffusion processes in the earth's crust into consideration.

$M-\log D$ relations suggest that a subdiscipline $g$ precursor represents a premonitory crustal strain of the order of $10^{-7}-10^{-6}$, while that for subdiscipline $r$ a strain of $10^{-9}-10^{-8}$. Precursors for other subdisciplines represent a strain of $10^{-8}-10^{-7}$. It is remarkable that precursors are sometimes observed at a point of which the epicentral distance is several times as large as that of epicentral area.
\end{abstract}

\section{Introduction}

That the magnetic field of the earth sometimes changes before an earthquake has long been recognized. It was reported, for example, that a huge horseshoe magnet had lost its attractive power immediately before the 1885 Edo (now Tokyo) earthquake of magnitude $(M) 6.9$ (MUSHA, 1951) although such a legend is hard to believe in view of modern knowledge of geomagnetism. MILNE (1890) presented a summary report on magnetic and electric phenomena related to earthquakes in early days.

In spite of much effort toward detecting changes in the geomagnetic field associated with an earthquake and possibly prior to an earthquake since such early days, it took a long time to firmly establish that changes in the geomagnetic field actually take place forerunning an earthquake on favourable occasions.

This is also the case for premonitory changes in the geoelectric field and earth resistivity. It can be said that reliable data of seismo-electricity have been put forward only recently.

An earthquake prediction programme of nation-wide scale has been underway in Japan since 1965, so that it has become possible to intensively search for earthquake 
precursors including magnetic and electric ones. EARTHQUAKE PREPAREDNESS Division, SHIZUOKA PREFECTURAL GOVERNMENT (1985) recently compiled earthquake precursor data taken by instrumental observations in Japan. The report provides the most up-to-date data-base of earthquake precursors in Japan. Many data covered by the report were taken by observations under the earthquake prediction programme.

In view of the data of considerable number as accumulated by now, it appears to the writer that an analysis of earthquake precursor data could be conducted in the hope of clarifying their physical nature. No comprehensive study on physical mechanism of earthquake precursor has so far been presented probably because of scarcity of observed data. This is especially so for magnetic and electric precursors of which reliable data are fewer than geodetic and seismic ones.

This paper aims at analyzing the data of magnetic and electric anomalies precursory to earthquakes so far obtained in Japan, although a more extensive study of earthquake precursors as a whole will be presented elsewhere.

A brief overview of the data set will be presented in Section 2. Section 3 is concerned with an analysis of precursor time $(T)$, the time interval between precursor appearance and main shock occurrence, mostly in relation to main shock magnitude $(M)$. As the $T-M$ relation is different from a group of subdisciplines to another group, a speculation possibly applied to interpreting such a difference will be put forward.

The relation between main shock magnitude $(M)$ and epicentral distance $(D)$ of a spot where a precursor is observed will be studied in Section 4. An interesting fact that clear-cut differences in $M-D$ relation are observed between subdisciplines of magnetic and electric precursors will be presented. It will be shown that precursory changes in the absolute value of geomagnetic field reflect crustal strain of the order of $10^{-7}-10^{-6}$, and that those in resistivity as observed by a particular resistivity variometer correspond to crustal strain of the order of $10^{-9}-10^{-8}$. As a result of the analyses of $T-M$ and $M-D$ relations, possible mechanism of magnetic and electric precursors, which may be physically acceptable, will be presented.

\section{Data}

Only the magnetic and electric data so far obtained in Japan are analyzed. The data were published by the EARTHQUAKe PrePAREDNESS Division, SHIZUOKA PREFECTURAL GOVERNMENT (1985) along with precursor data of geodetic, seismic and geochemical disciplines.

As it is tedious to describe all the data, only the numbers of data are given in Table 1 for each subdiscipline of magnetic and electric precursor. Those who are particularly interested in the detail of those precursors are kindly requested to refer to the original report although the writer is afraid that it is written solely in Japanese.

Geomagnetic field change (subdiscipline 1: abbreviation $g$ ) means change in the geomagnetic field as detected by repeated magnetic surveys and/or continuous geomagnetic observations at a fixed observation point. In association with the introduction of proton precession magnetometer, highly reliable data with an 
Table 1. The number of data for electric and magnetic precursors in Japan.

\begin{tabular}{clcc}
\hline No. & \multicolumn{1}{c}{ Subdiscipline } & Abbreviation & Number of data \\
\hline 1 & Geomagnetic field & $g$ & 5 \\
2 & Earth currents & $e$ & 10 \\
3 & Resistivity 1 & $r$ & 30 \\
4 & Resistivity 2 & $R$ & 9 \\
5 & Electromagnetic radiation & $w$ & 7 \\
\hline \multicolumn{2}{r}{} & & Total
\end{tabular}

accuracy of $1 \mathrm{nT}$ or so has been available since 1960s.

Earth currents (subdiscipline 2: $e$ ) is usually represented by differences in electric potential between two electrodes buried in the ground.

There are two ways for measuring earth resistivity. For subdiscipline $3(r)$ or "resistivity 1", a resistivity variometer basing on the so-called four-pole method is used for continuous measurement. The electrode distance is only 1-2 m. Precursory changes of this kind observed at a station about $60 \mathrm{~km}$ southwest of Tokyo are analyzed (e.g., RIKITAKE and YAMAZAKI, 1985). Meanwhile, resistivity change as disclosed by the so-called dipole-dipole or Schlumberger methods is denoted by "resistivity 2 " and subdiscipline $4(R)$. Electrode distances usually amount to several tens or hundreds of meters in this case. Change in transfer function for short-period geomagnetic variations is assumed to belong to this subdiscipline as well.

It has recently been reported that electromagnetic radiation of wide band is sometimes observed preceding an earthquake although no physical nature of such a phenomenon has as yet been worked out. This is subdiscipline $5(w)$.

In order to demonstrate what magnetic and electric precursors are like, only three typical examples are shown in the following. Figure 1 (SASAI and ISHIKAWA, 1980) shows the change in the geomagnetic total intensity at Kawazu station in Izu Peninsula. A change amounting to $5 \mathrm{nT}$ or thereabout foreran a magnitude 5 earthquake that occurred at an epicentral distance of $4 \mathrm{~km}$ or so.

A marked anomaly in earth potential was observed by MIYAKOSHI (1985) preceding an earthquake of $M 5.6$ that occurred on the Yamasaki fault, an outstanding active fault in the Chugoku district about $100 \mathrm{~km}$ west of Kyoto, in June, 1984 as shown in Fig. 2. In addition to the anomalous change shown in the figure, it was reported that some anomalously large fluctuations of earth potential had been taking place since several tens of days before the shock.

An unusually sensitive resistivity variometer (YAMAZAKI, 1967, 1968) has been in operation at a sea-shore observation point about $60 \mathrm{~km}$ southwest of Tokyo. Anomalous changes in earth resistivity have often been observed by the variometer several hours preceding a conspicuous earthquake. It is remarkable that such a premonitory change is observed in association with an earthquake of which the epicentral distance amounts to $1000 \mathrm{~km}$ or thereabout provided the earthquake magnitude amounts to 8 or so. Figure 3 shows an example of resistivity precursor that 

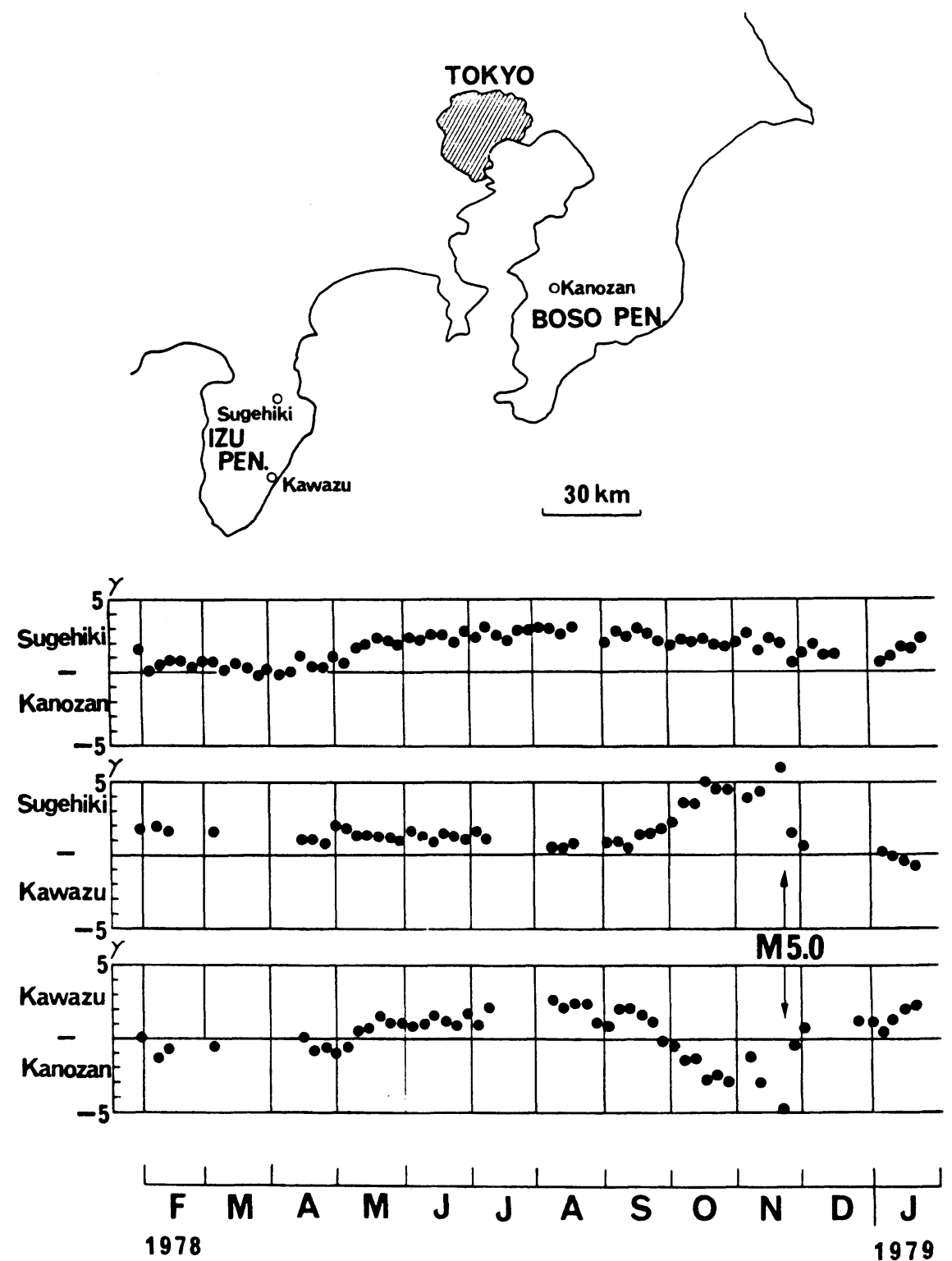

Fig. 1. Changes in the difference in the 5-day means of geomagnetic total intensity in units of nT between the three stations; Kawazu, Sugehiki and Kanozan (SASAI and IsHIKAWA, 1980). 


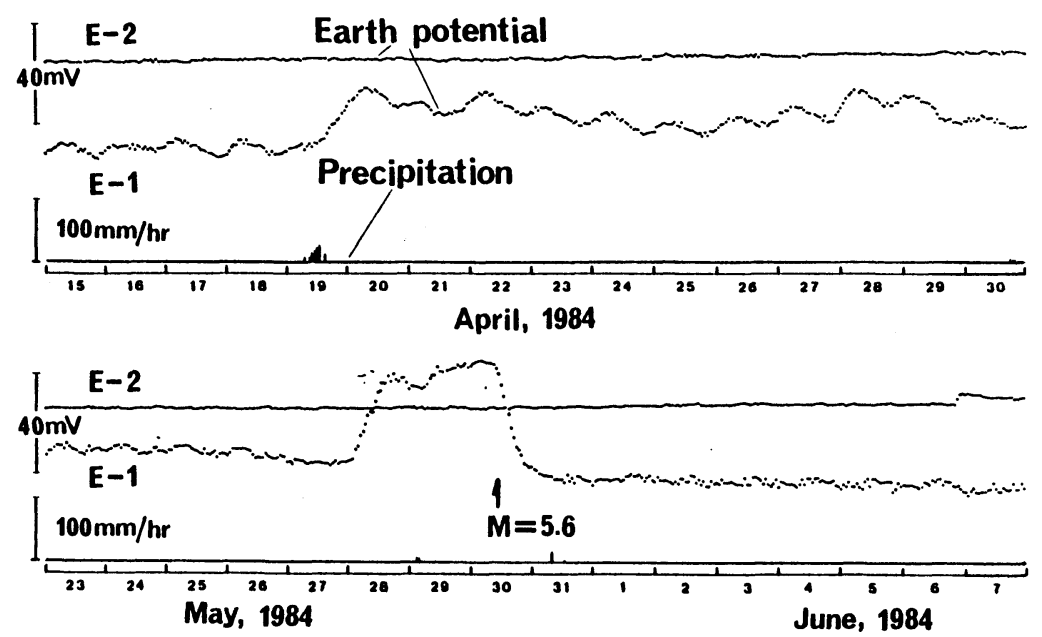

Fig. 2. Changes in earth potential preceding an earthquake of $M 5.6$ on the Yamasaki fault. The epicentral distance is about $3 \mathrm{~km}$. (MIYAKOSHI, 1985).

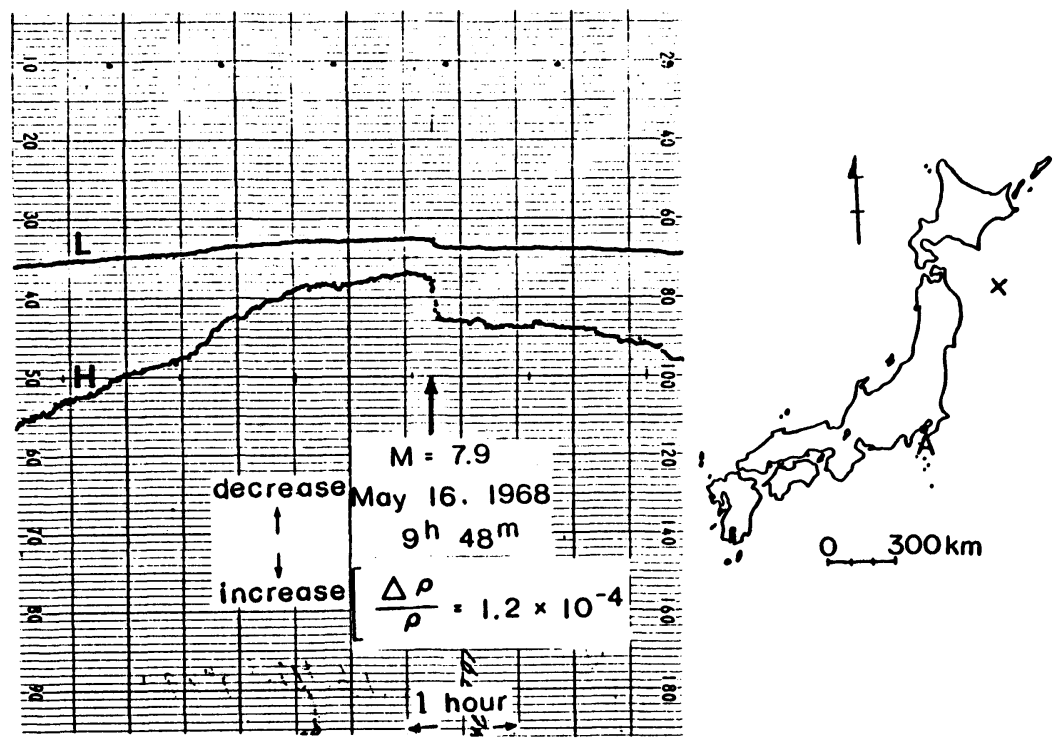

Fig. 3. Low (L) and high ( $\mathrm{H})$ sensitivity records of resistivity change at Aburatsubo in association with the Tokachi Oki earthquake of $M 7.9$ (RIKITAKE and YAMAZAKI, 1970). 
was observed in association with the Tokachi Oki earthquake of M7.9 in May, 1968 (RIKITAKE and YAMAZAKI, 1970).

3. Precursor Time $(T)$ and Main Shock Magnitude $(M)$ Relation

RIKITAKE (1975) was the first, who collected then-available world-wide data of earthquake precursors and examined the relation between precursor time $(T)$ and main shock magnitude $(M)$. HoNKURA (1981) further investigated into such a relation especially for magnetic and electric precursors.

As the number of available data has considerably increased, it is of particular interest to reexamine the $T-M$ relation of magnetic and electric precursors on the basis of the present data set. In Figs. 4, 5, 6, 7 and 8 are shown the $\log T-M$ plots for subdisciplines $g, e, R, r$ and $w$, respectively. Precursor time $(T)$ is measured in units of day and $\log T$ is used for the plots. As can be seen in the figure, it seems likely that $\log T$ is correlated approximately linearly to $M$ for subdisciplines $g, e$ and $R$, and that the larger the main shock magnitude is, the longer is the precursor time. On the contrary, no such relation is observed for subdisciplines $r$ and $w$. Rather, it seems that precursor times are distributed around $\log T=-1$ independently of main shock magnitude.

Following RIKITAKE $(1976,1979)$, precursors of the former three disciplines may be called the precursor of the first kind for which the following relation

$$
\log T=a+b M
$$

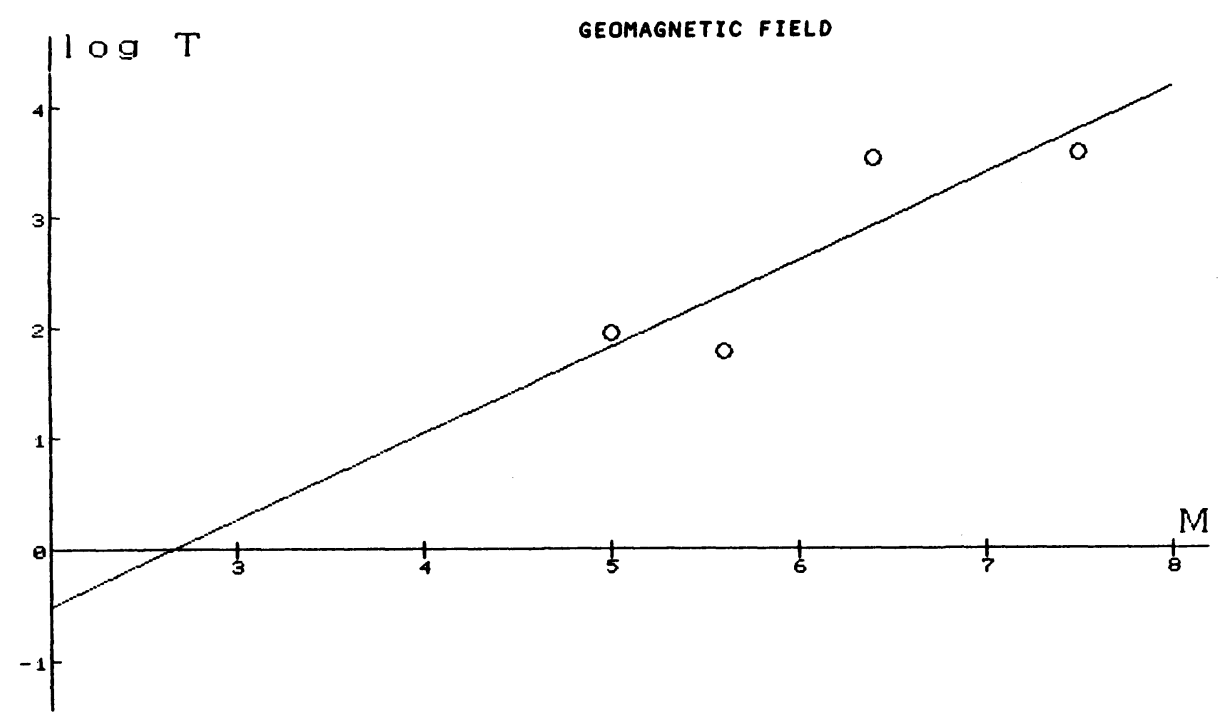

Fig. 4. $\log T-M$ relation for subdiscipline $g$ (geomagnetic field). 


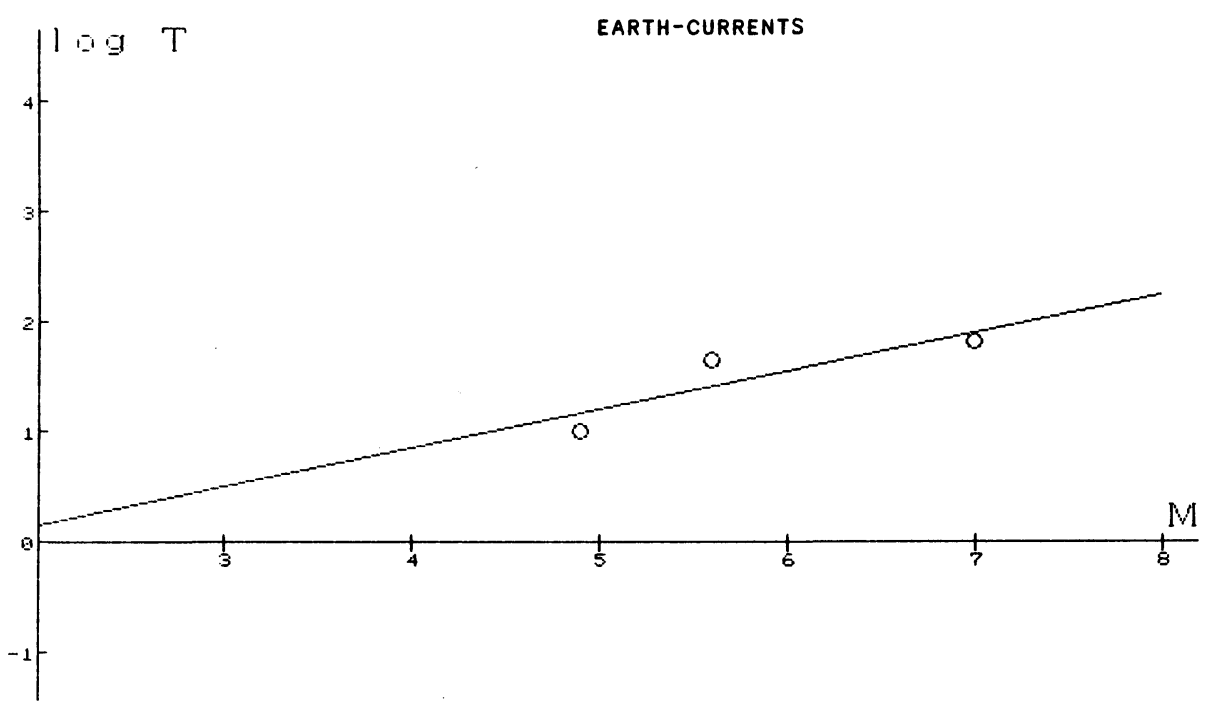

Fig. 5. $\log T-M$ relation for subdiscipline $e$ (earth currents).

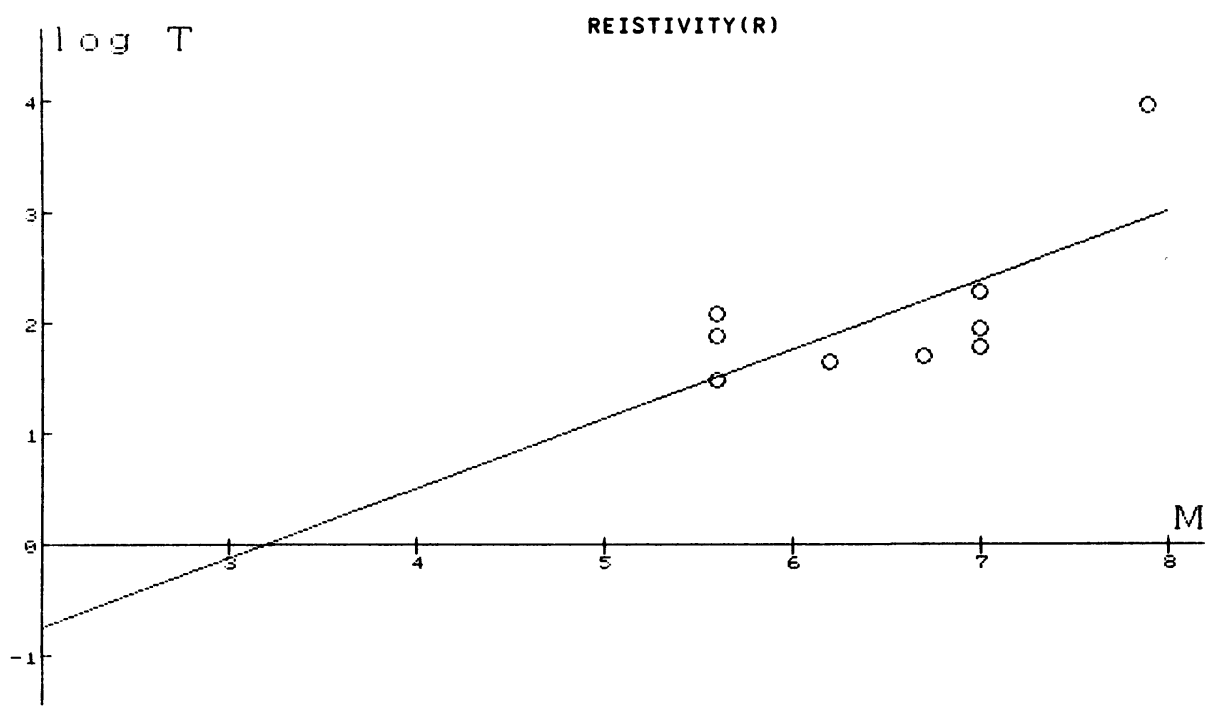

Fig. 6. $\log T-M$ relation for subdiscipline $R$ (resistivity 2 ). 


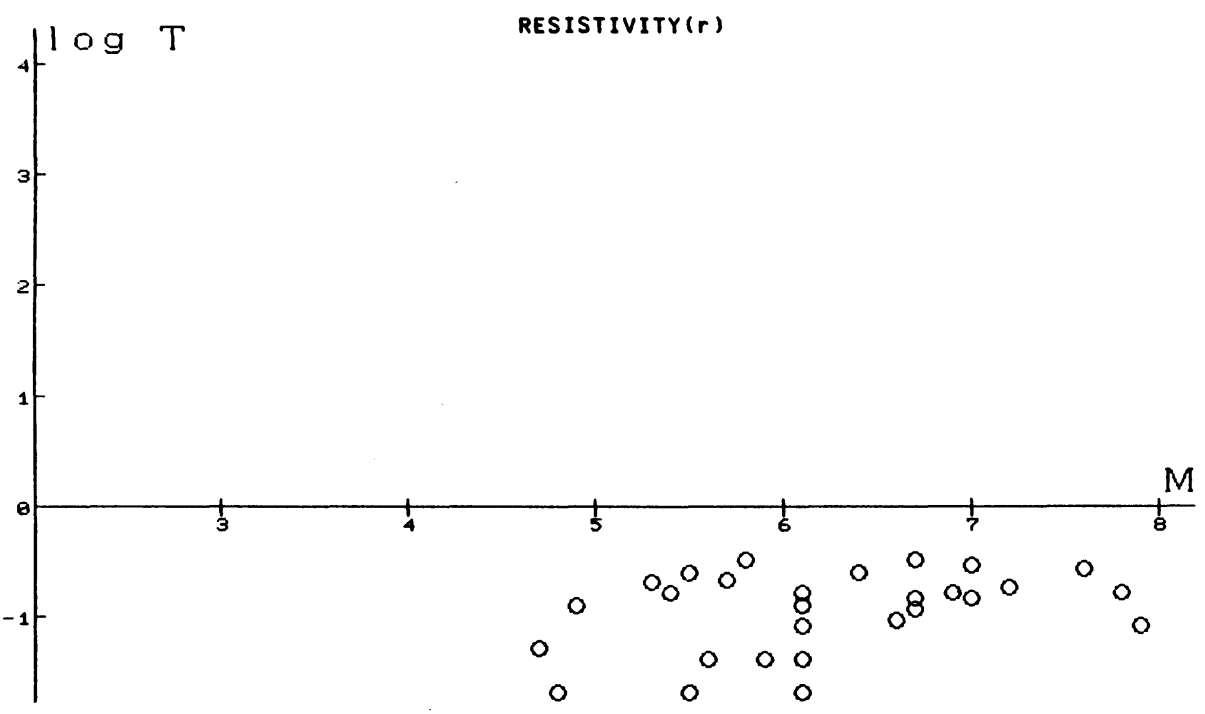

Fig. 7. $\log T-M$ relation for subdiscipline $r$ (resistivity 1).

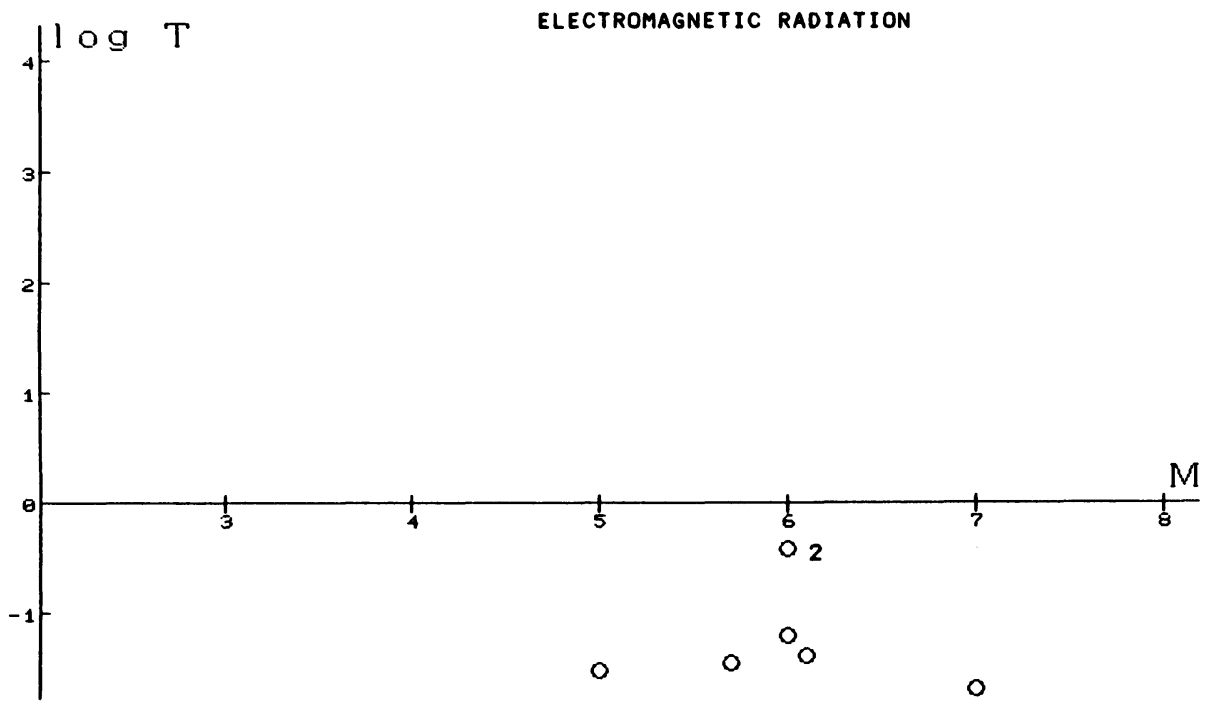

Fig. 8. $\log T-M$ relation for subdiscipline $w$ (electromagnetic radiation). 
may be approximately held. Parameters $a$ and $b$ as determined by the least squares method for subdisciplines $g, e$ and $R$ are given in Table 2 .

It is noticed, however, that precursor time for discipline $g$ is significantly larger than that for disciplines $e$ and $R$. At this point, it is interesting to compare the above-obtained $T-M$ relations to those of precursors other than magnetic and electric signals. For example, Figure 9 shows the $\log T-M$ relation for land deformation (discipline: $l$ ) as obtained by geodetic means and tide-gauge observation. Logarithmic precursor time for magnitudes ranging 5-7 indicated by the best-fitting straight line seems likely to roughly coincide with that for discipline $g$. The fact therefore suggests that precursors for disciplines $l$ and $g$ may be brought about by the same cause. Meanwhile, precursors for disciplines $e$ and $R$ may be caused by some other mechanism.

Table 2. Parameters $a$ and $b$ involved in Eq. (1) and their probable errors as determined by the least squares method.

\begin{tabular}{lccc}
\multicolumn{1}{c}{ Subdiscipline } & Abbreviation & $a$ & $b$ \\
\hline Geomagnetic field & $g$ & $-2.08 \pm 1.43$ & $0.78 \pm 0.23$ \\
Earth currents & $e$ & $-1.06 \pm 0.52$ & $0.44 \pm 0.08$ \\
Resistivity 2 & $R$ & $-2.00 \pm 1.16$ & $0.63 \pm 0.18$ \\
\hline
\end{tabular}

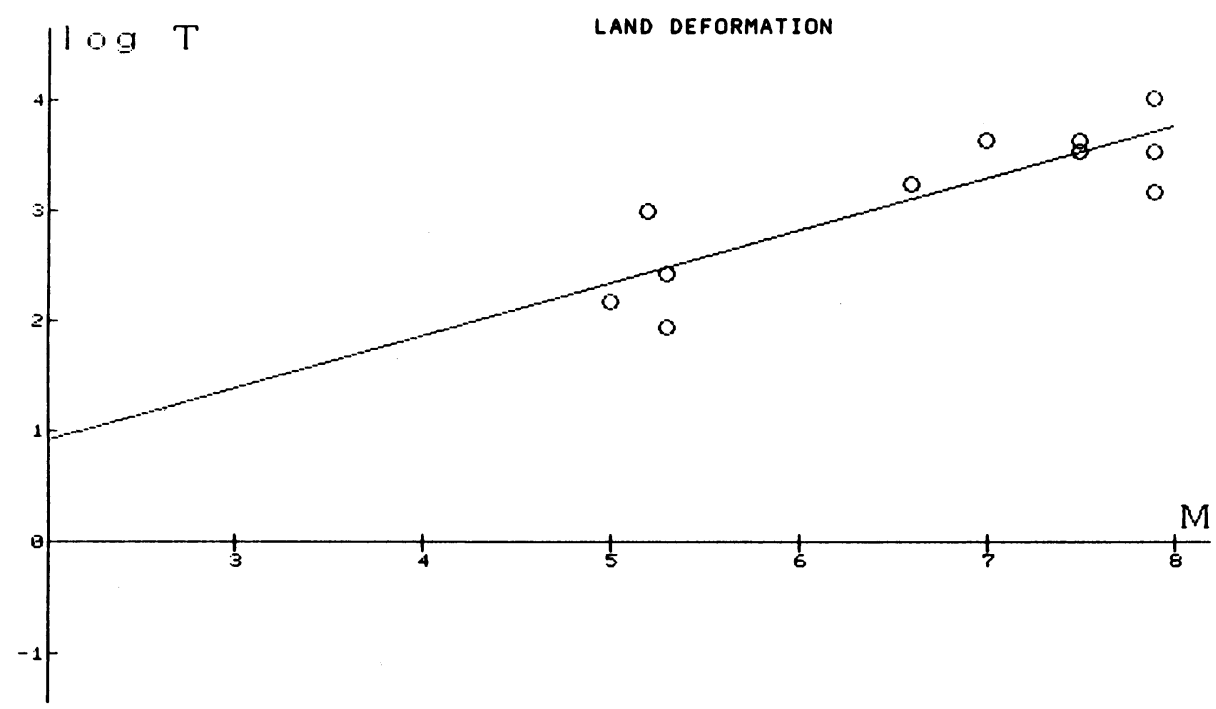

Fig. 9. $\log T-M$ relation for discipline $l$ (land deformation) as observed by geodetic means and tide-gauge observation. 
In light of the characteristics of magnetic and electric precursors of the first kind, the writer proposes the following speculation on the possible mechanism of precursor appearance. When crustal stress becomes high, land deformation such as anomalous uplift may take place perhaps because of generation of dilatancy. At the same time or a little in advance, a change in the geomagnetic field due to piezomagnetic effect may possibly be observed. It is not unreasonable, therefore, to expect that precursors for disciplines $l$ and $g$ are observed approximately at the same time.

In the next place, diffusion of underground water into the dilatant portion of the earth's crust may well be expected. Such a diffusion process may give rise to changes in earth resistivity and so earth currents. It may necessarily take some time to complete such diffusion, so that precursors for disciplines $e$ and $R$ may appear some time later. Close examination of the present data-set indicates that such a delay of precursor appearance is also confirmed for precursors of underground water and radon concentration.

That the $T-M$ relation of magnetic and electric precursors is complicated when precursors of all subdisciplines are analyzed at the same time has been pointed out by HONKURA (1981). He also suggested that it is the longest precursor time that should be controlled by the dilatancy-diffusion process. The above speculation seems to support Honkura's view.

Precursors for disciplines $r$ and $w$, which apparently show no magnitude dependence of precursor time, are called the precursors of the second kind. Nothing definite about the physical mechanism of this class of precursor is known although it is speculated that they are associated with preliminary rupture process in the focal region immediately prior to main shock occurrence. RIKITAKE and YAMAZAKI (1985) showed that a relation such as

$$
\log T=\alpha M+\beta \log R
$$

seems likely to hold good for precursors for discipline $r$, where $R$ is the hypocentral distance and $\alpha$ and $\beta$ are constants. It is then speculated that Eq. (2) can be deduced by assuming a rupture propagation in the focal region and a suitable diminishing rate of signal depending on the distance from the focal region.

\section{Main Shock Magnitude $(M)$ and Epicentral Distance $(D)$ Relation}

It is important for practical earthquake prediction to know the limit of epicentral distance for which a precursor is detected. Taking logarithmic epicentral distance $(D)$ in $\mathrm{km}$ as the abscissa and main shock magnitude $(M)$ as the ordinate, $M$-log $D$ plots for precursors of magnetic and electric subdisciplines are shown in Figs. 10, 11, 12 and 13. Looking at these figures, we see that the larger the main shock magnitude is, the larger is the detectable distance from the epicenter. Although such a tendency can be seen for all the subdisciplines, the straight lines which represent general scattering of $M-\log D$ plots seem to be different from subdiscipline to subdiscipline. It is clear that the $M-\log D$ plot for subdiscipline $g$ lies to the most left on the $M-\log D$ plane. 


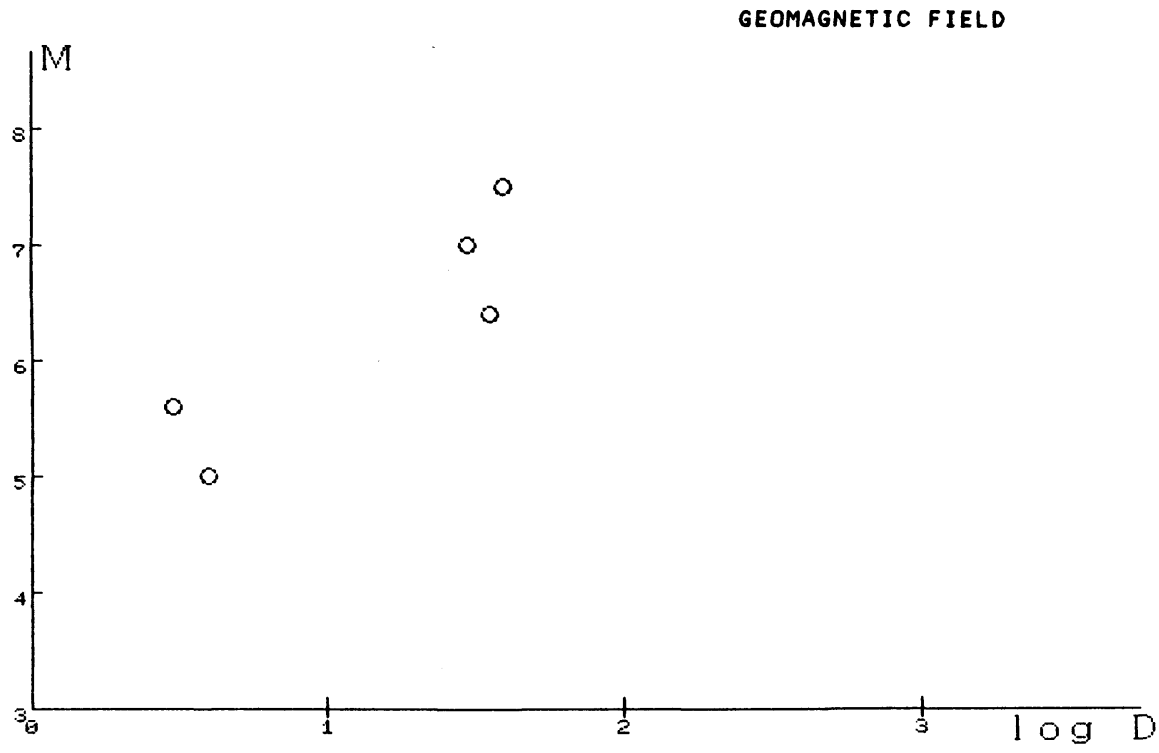

Fig. 10. $M-\log D$ relation for subdiscipline $g$ (geomagnetic field).

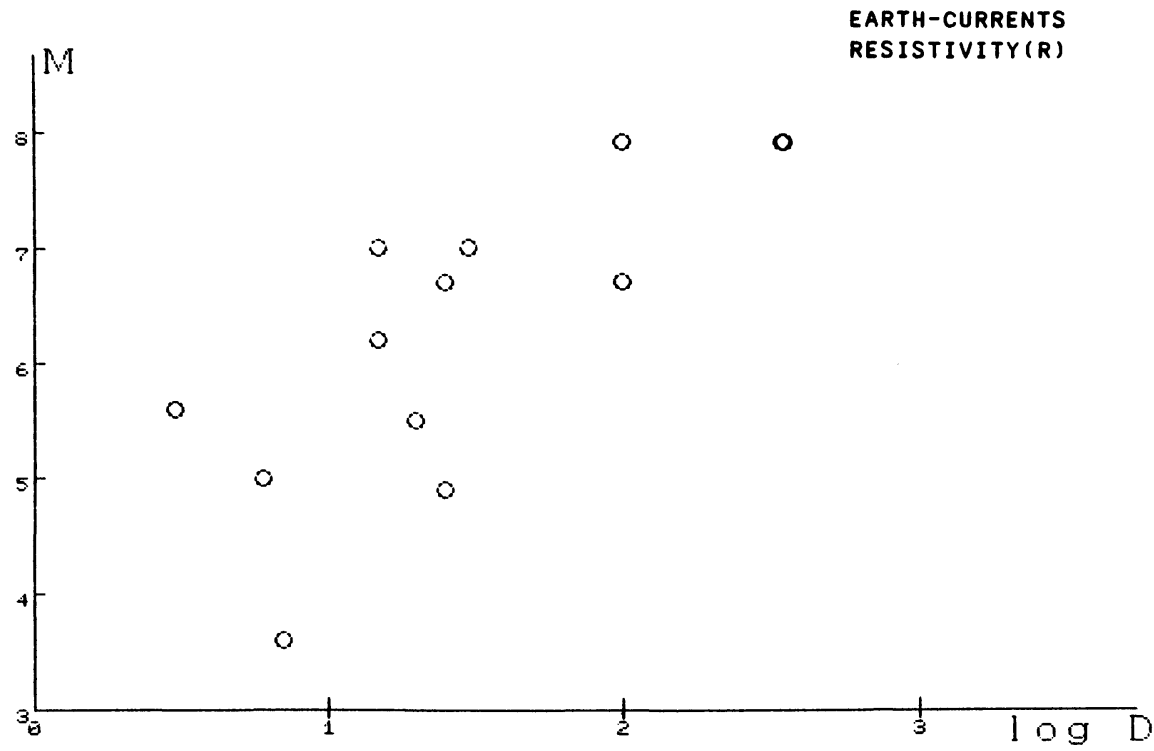

Fig. 11. $M-\log D$ relation for subdisciplines $e$ (earth currents) and $R$ (resistivity 2 ). 


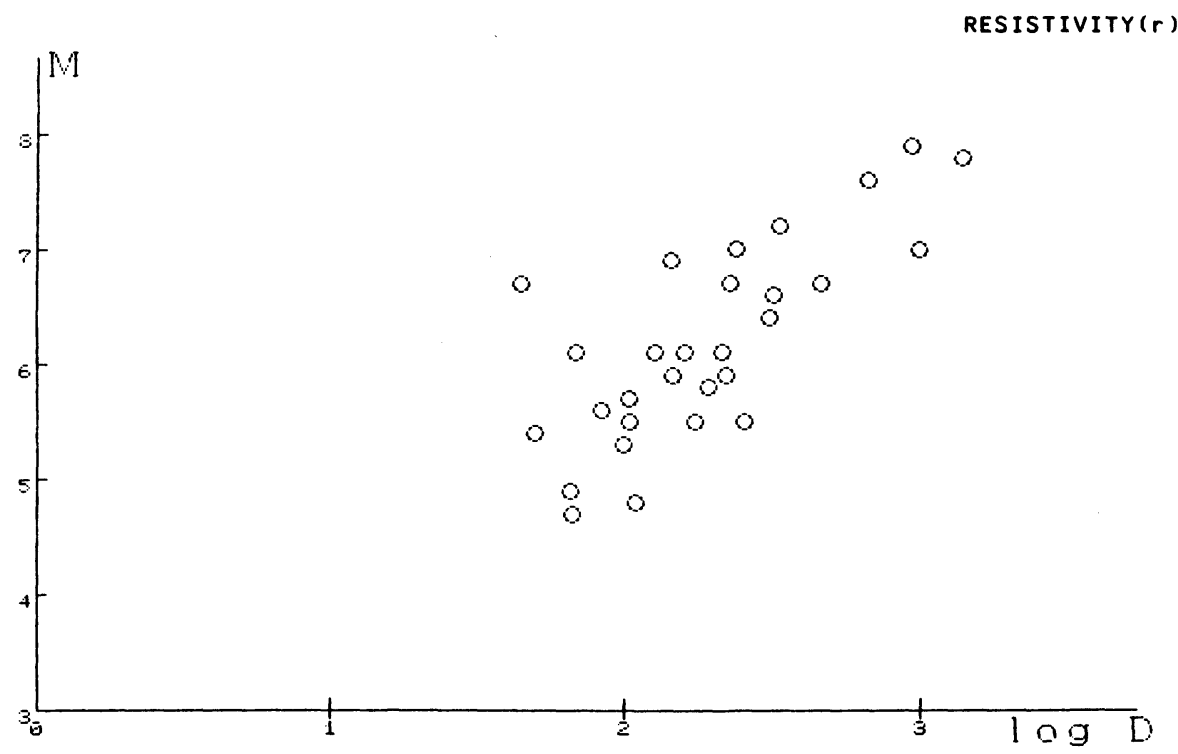

Fig. 12. $M-\log D$ relation for subdiscipline $r$ (resistivity 1 ).

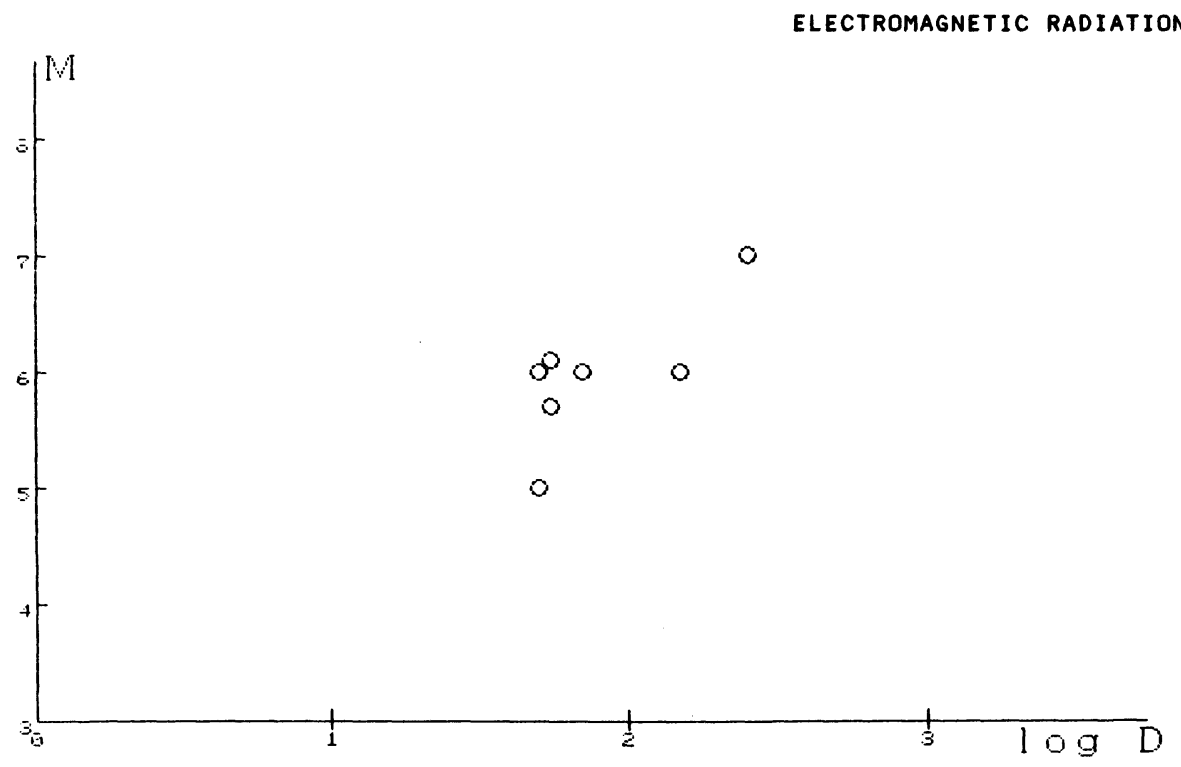

Fig. 13. $M-\log D$ relation for subdiscipline $w$ (electromagnetic radiation). 
Meanwhile, that for subdiscipline $r$ is located to the most right. And those for other magnetic and electric subdisciplines seem to scatter between the above-mentioned two plots.

It is of course possible to have similar plots for precursors other than magnetic and electric disciplines. The plot for discipline $l$ or land deformation seems to roughly agree with that for discipline $g$, while the plot for discipline $r$ is located at the most right portion of $M-\log D$ plots for all disciplines.

WIDEMAN and MAJOR (1967) and TAKEMOTO (1970), who analyzed coseismic strain steps associated with earthquakes, presented curves for which a strain step of specified amplitude is to be observed on the $M-\log D$ plane on an empirical basis. As long as we assume that precursors are associated with distant strain field caused by some crustal effects at the focal region premonitory to an earthquake, it is not unreasonable to assume that the $M-\log D$ plots for precursors reflect the strain state.

In Fig. 14 are shown all the $M-\log D$ plots for the present precursor data-set including those other than magnetic and electric precursors. In the figure, straight lines that approximately represent coseismic steps amounting to $10^{-9}-10^{-6}$ in strain are also shown. A simple model assuming an underground spherical cavity of high

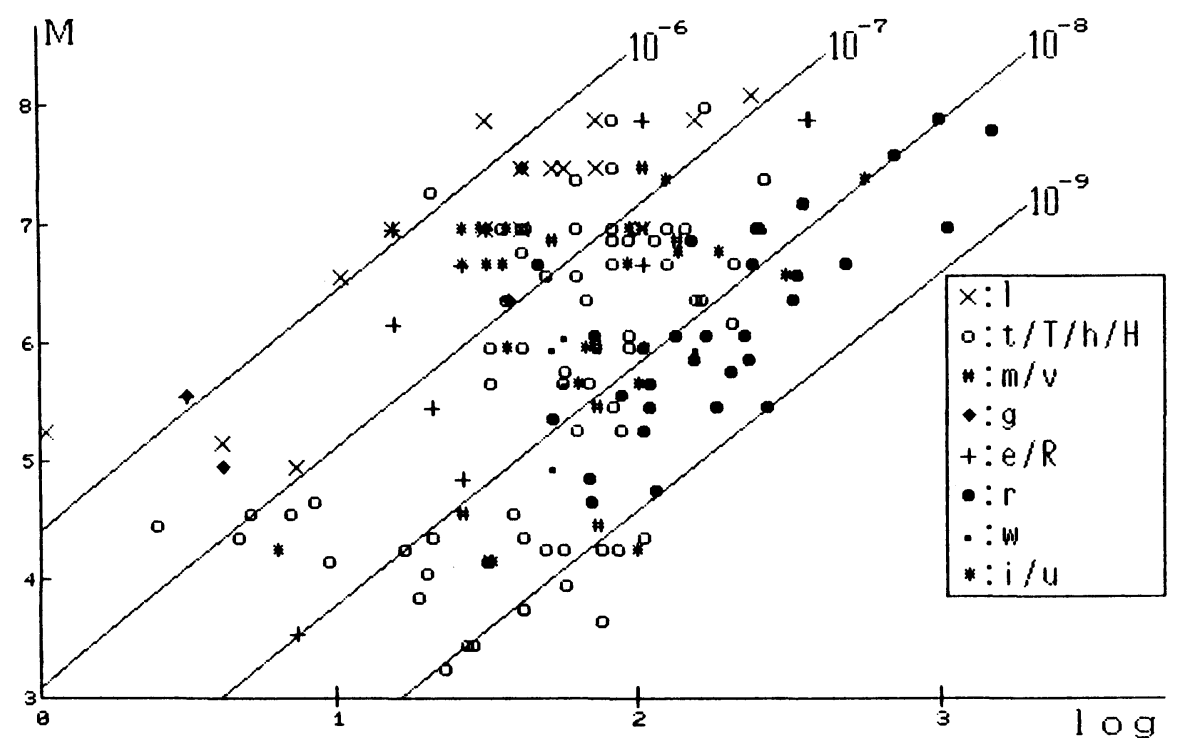

Fig. 14. $M-\log D$ plots for earthquake precursors of all available disciplines. Abbreviations $l, t, T, h, H$, $m, v, g, e, R, r, w, i$ and $u$ denote disciplines land deformation, tilting of the ground observed by a pendulum tiltmeter, tilting by a water-tube tiltmeter, crustal strain by an extensometer, strain by a dilatometer, microearthquake, change in seismic wave velocity, geomagnetic field, earth currents, resistivity 2 , resistivity 1 , electromagnetic radiation, radon concentration and underground water/ hot spring, respectively. Approximate straight lines along which crustal strain due to focal process takes on a specified value are shown. 
pressure leads to similar distribution of equal strain lines although the detail of such a study is not presented here.

Looking at Fig. 14, we may surmise that precursors for discipline $l$ reflects a precursory strain amounting to the order of $10^{-7}-10^{-6}$. This is also the case for precursors for subdiscipline $g$. Meanwhile, precursors for subdiscipline $r$ correspond to a strain of the order of $10^{-9}-10^{-8}$. Precursors for other magnetic and electric subdisciplines cover a strain state approximately amounting to $10^{-8}-10^{-7}$.

\section{Discussion and Conclusions}

The analysis of $M-\log D$ relation of magnetic and electric precursors as presented in the last section suggests that these precursors are closely connected to strain state in the earth's crust premonitory to earthquake occurrence. It is further indicated that the sensitivity of precursor in terms of crustal strain is different from subdiscipline to subdiscipline. Precursors belonging to subdiscipline $r$ seem likely to be most sensitive to crustal strain forerunning an earthquake, a precursor of this subdiscipline being able to represent a crustal strain of the order of $10^{-9}-10^{-8}$. It is therefore possible to observe a precursor for subdiscipline $r$ at an observation point of which the epicentral distance amounts to $1000 \mathrm{~km}$ or so provided the main shock magnitude amounts to 8 or over.

It is believed that seismic energy is stored in an epicentral region of which the mean diameter is about $100 \mathrm{~km}$ or thereabout for an earthquake of magnitude 8 . It is also believed that earthquake precursors, if any, may be observed mostly over such an epicentral area. The present study of $M-\log D$ relation indicates, however, that a precursor could be observed even at an epicentral distance several times larger than the effective radius of epicentral area on favourable cases.

It is interesting to note that a precursor of subdiscipline $g$ or change in the geomagnetic field reflects a crustal strain state equivalent to that revealed by land deformation which is believed to be most reliable among various disciplines of earthquake precursor. A premonitory strain represented by this kind of precursor amounts to $10^{-7}-10^{-6}$, the largest value of strain for a precursor. Premonitory strains monitored by precursors of subdiscipline $g$, which is seemingly equivalent to discipline $l$, being not quite small, such a signal may be highly reliable once it is correctly detected.

As mentioned in Section 3, the analysis of $\log T-M$ relation brought out a possible process of earthquake generation in a focal region. For a preseismic period during which crustal stress is built up, we may be able to expect a signal of subdiscipline $g$ probably due to piezomagnetic effect. It is not unreasonable to account for subsequent appearance of precursors of subdisciplines $e$ and $R$ basing on the dilatancy-diffusion process. The difference in precursor time between precursors for different subdisciplines may thus be explained.

Scattering of $\log T-M$ plots is considerable when magnetic and electric precursors of all subdisciplines are plotted at the same time as pointed out by HONKURA (1981). In view of the analysis in Section 3, however, the reason why we 
have such an apparently irregular distribution can be ascribed to the fact that $\log T-M$ relations are different from subdiscipline to subdiscipline of magnetic and electric precursor.

As for precursors of subdisciplines $r$ and $w$, which are classified into precursors of the second kind, nothing remarkable for clarifying their physical nature is obtained by this study.

Most data analyzed in this paper are collected by Professor Y. Honkura. The writer is grateful to him for his effort.

\section{REFERENCES}

Earthquake Preparedness Division, Shizuoka Prefectural Government, Present Status of the Analysis of Earthquake Precursors, 146pp., 1985 (in Japanese).

HoNkURA, Y., Electric and magnetic approach to earthquake prediction, in Current Research in Earthquake Prediction I, edited by T. Rikitake, pp. 301-383, Center for Academic Publications Japan (Tokyo), D. Reidel Publishing Company, Dordrecht, 1981.

MILNE, J., Earthquakes in connection with electric and magnetic phenomena, Trans. Seismol. Soc. Japan, $15,135-162,1890$.

MiYakoshi, J., On some problems of the variation of self-potentials observed in an active fault, the Yamasaki fault, Disaster Prev. Res. Inst. Annuals, 28B, 127-132, 1985 (in Japanese).

Musha, K., Nihon Jishin Shiryo (Japanese Historical Records Relevant to Earthquakes), 1019pp., Mainichi Press, Tokyo, 1951 (in Japanese).

RIKITAKE, T., Earthquake precursors, Bull. Seismol. Soc. Am., 65, 1133-1162, 1975.

Rikitake, T., Earthquake Prediction, 357pp., Elsevier, Amsterdam, 1976.

RIKITAKE, T., Classification of earthquake precursors, Tectonophysics, 54, 293-309, 1979.

RikitaKe, T. and Y. YAMAZAKı, Strain steps as observed by a resistivity variometer, Tectonophysics, 9 , 197-203, 1970.

Rikitake, T. and Y. Yamazakı, The nature of resistivity precursor, Earthq. Predict. Res., 3, 559-570, 1985.

SASAI, Y. and Y. ISHIKAWA, Tectonomagnetic event preceding a M 5.0 earthquake in the Izu peninsula -a seismic slip of a buried fault?, Bull. Earthq. Res. Inst., Univ. Tokyo, 55, 895-911, 1980.

Tакемото, S., Strain steps and the dislocation fault model, Bull. Disas. Prev. Res. Inst., Kyoto Univ., 20, $1-15,1970$.

Wideman, C. J. and M. W. MAJOR, Strain steps associated with earthquakes, Bull. Seismol. Soc. Am., 57, 1429-1444, 1967.

YAMAZAKI, Y., Electrical conductivity of strained rocks (3rd paper), A resistivity variometer, Bull. Earthq. Res. Inst., Univ. Tokyo, 45, 849-860, 1967.

YAMAZAKI, Y., Electrical conductivity of strained rocks (4th paper), Improvement of the resistivity variometer, Bull. Earthq. Res. Inst., Univ. Tokyo, 46, 957-964, 1968. 